\title{
Fiber Bragg grating fabrication by femtosecond laser radiation
}

\author{
Anton Chernikov*, Dmitriy Kochuev, Kirill Khorkov, Ruslan Chkalov, and \\ Nikolay Davydov \\ Vladimir State University, 600000 Vladimir, Russia
}

\begin{abstract}
The paper presents the results of fiber Bragg gratings fabrication by femtosecond laser radiation using point-by-point and lineby-line inscription methods. The approach makes it possible to fabricate the second and higher diffraction orders fiber Bragg gratings, which can be used as sensitive elements of fiber-optic sensors.
\end{abstract}

\section{Introduction}

In the last decade, the field of research related to the formation of optical structures in the bulk of transparent dielectrics, including optical fibers, using femtosecond laser radiation is very promising. The action of laser radiation leads to a local modification of the refractive index of the regions that were exposed, thus, ordered structures of the refractive index are formed. These structures are fiber Bragg gratings (FBGs), which are widely used in various fiber-optic devices: as sensitive elements in sensors [1,2], as spectral filters in fiber lasers $[3,4]$, etc.

\section{Fiber Bragg grating fabrication by femtosecond laser radiation}

The FBG was recorded using $\mathrm{Yb}: \mathrm{KGW}$ femtosecond laser system operating at wavelength of $1030 \mathrm{~nm}$, pulse width $280 \mathrm{fs}$, pulse repetition rate of $10 \mathrm{kHz}$. Laser radiation was focused using a Mitutoyo Plan Apo NIR high-aperture micro lens $(100 x, N A=0.7)$. For moving of the fiber during inscription high-precision 2-dimensional positioning system Aerotech ANT130-110-XY Ultra and motorized linear stage Standa 8MT167-25LS (Z-axis) were used. For inscription of FBGs standard single mode fiber Corning SMF-28e+ was used (core diameter $8.2 \mu \mathrm{m}$ ), FBGs were inscribed according to the method of line-by-line and point-by-point technology at an energy of $150 \mathrm{~nJ}$ through the polymer jacket. The period of FBGs was chosen taking into account the second, third and fourth diffraction orders based on the linear dimensions of the inscribed structures.

Inscription of FBGs was done by translating the fiber regarding to the focused laser beam. For precise flatness position adjustment of the sample the tilt corrector system (Standa 8MKVDOM) was used.

To overcome the fabrication limitation imposed by the intrinsic fiber geometry, the

*Corresponding author: an4ny.che@gmail.com 
optical fiber was placed between slide and cover glass, space between them filled with index-matching immersion liquid with a refractive index close to the refractive index of optical fiber. Glycerin had been used as the immersion liquid [5].

Micrographs of fourth order FBG (period $\Lambda=2.14 \mu \mathrm{m}$, central Bragg wavelength 1550 $\mathrm{nm}$ ) inscribed using point-by-point fabrication method by femtosecond laser radiation is shown in Fig. 1.

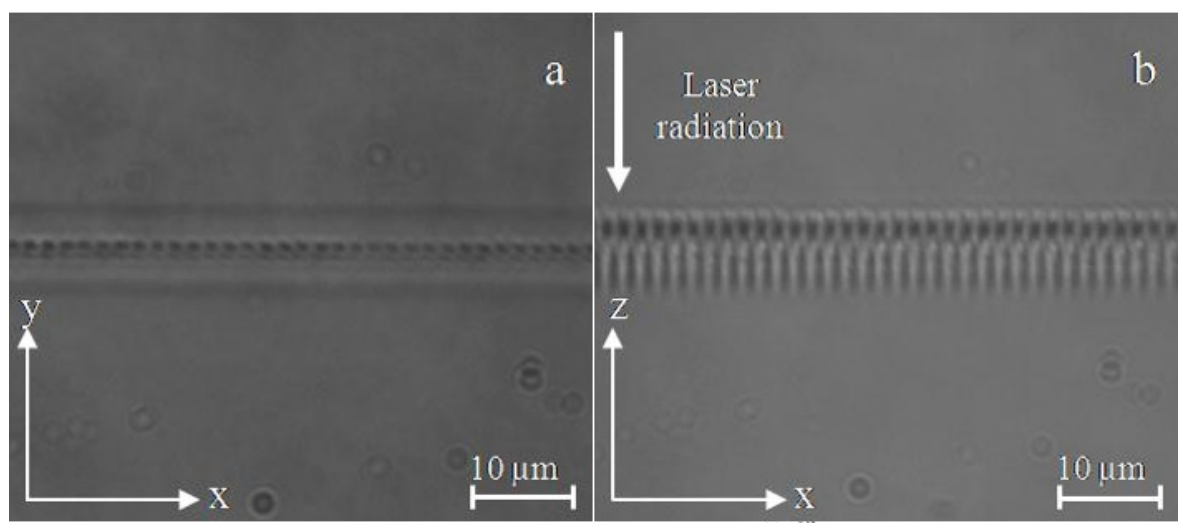

Fig. 1. Micrographs of fourth order FBG inscribed using point-by-point fabrication method: a - top view; $\mathrm{b}$ - orthogonal view.

Fig. 1 shows that the modification region completely intersects core of the optical fiber and does not deviate from its initial position along the $\mathrm{Z}$ axis. FBG fabricated using point-bypoint inscription method is a set of individual cylinders.

In the case of line-by-line inscription method the formation of FBG is carried out sequentially in the form of strokes, this method is less demanding to configure the system. The main disadvantage of this method is the rather low recording speed due to the fact that it is necessary to modify large-sized areas. Using the line-by-line inscription method were inscribed the second $(\Lambda=1.07 \mu \mathrm{m})$, third $(\Lambda=1.605 \mu \mathrm{m})$ and fourth diffraction order $(\Lambda=1.605 \mu \mathrm{m})$ FBGs. Reflection spectrum of inscribed FBG were performed using optical spectrum interrogator module (OSI) NI PXIe-4844.

\section{Conclusion}

The paper presents the results of fabrication the second and higher diffraction orders FBGs by femtosecond laser radiation using point-by-point and line-by-line inscription methods. Inscribed FBGs can be used as sensitive elements of fiber optic sensors.

\section{References}

1. K. O. Hill, Y. Fujii, D. C. Johnson, B. S. Kawasaki, Applied physics letters, 32, (1978).

2. C B Schaffer, A Brodeur, E Mazur, Measurement Science Technology, 12, (2001).

3. Dostovalov A V, Wolf A A and Babin S A. Quantum Electronics, 45, (2015).

4. Vasil'ev S A, Medvedkov O I, Korolev I G, Bozhkov A S, Kurkov A S and Dianov E M Quantum Electronics, 35, (2005).

5. Chernikov A S, Khorkov K S, Kochuev D A, Chkalov R V, Prokoshev V G and Davydov N N J. Phys.: Conf. Ser. 1164 012015, (2019). 